Proceedings of the 2007 Winter Simulation Conference

S. G. Henderson, B. Biller, M.-H. Hsieh, J. Shortle, J. D. Tew, and R. R. Barton, eds.

\title{
REPLICATED BATCH MEANS FOR STEADY-STATE SIMULATIONS WITH INITIAL TRANSIENTS
}

\author{
Christos Alexopoulos \\ Sigrún Andradóttir \\ H. Milton Stewart School of \\ Industrial and Systems Engineering \\ Georgia Institute of Technology \\ Atlanta, GA 30332-0205, U.S.A.
}

\author{
Nilay Tanık Argon \\ Department of Statistics and Operations Research \\ University of North Carolina \\ Chapel Hill, NC 27599-3180, U.S.A.
}

David Goldsman

H. Milton Stewart School of

Industrial and Systems Engineering

Georgia Institute of Technology

Atlanta, GA 30332-0205, U.S.A.

\begin{abstract}
We provide asymptotic expressions for the expected value and variance of the replicated batch means variance estimator when the stochastic process being simulated has an additive initial transient. These expressions explicitly show how the initial transient and the autocorrelation in the data affect the performance of the estimator. We apply our results to study how many replications will minimize the asymptotic bias of the variance estimator for a simple example.
\end{abstract}

\section{INTRODUCTION}

In simulation studies, it is often of interest to construct a confidence interval on the steady-state mean $\mu$ of a stationary output process $\left\{X_{k}\right\}_{k=1}^{\infty}$ (e.g., customer waiting times in a queueing system). The usual point estimator for $\mu$ is the sample mean based on $n$ observations, $\bar{X}_{n} \equiv \sum_{k=1}^{n} X_{k} / n$. The more-challenging task in constructing a confidence interval is estimating the variance of the sample mean, or equivalently, $n \operatorname{Var}\left(\bar{X}_{n}\right)$. When the number of observations is large, then the experimenter can instead estimate the variance parameter $\sigma^{2} \equiv \lim _{n \rightarrow \infty} n \operatorname{Var}\left(\bar{X}_{n}\right)$.

Two of the most popular methods for estimating $\sigma^{2}$ are the methods of independent replications (IR) and batch means (BM) (see, e.g., Fishman 2001). With the IR method, $r$ independent runs are conducted, which are used in calculating $r$ replicate sample means, each based on, say, $m$ observations. Then, $\sigma^{2}$ is estimated by $m$ times the sample variance of the replicate means. With the BM method, on the other hand, a single, long run is conducted, which is divided into $b$ contiguous batches, each of length, say, $m$. Then, the resulting batch sample means are computed and $\sigma^{2}$ is estimated by $m$ times the sample variance of the batch means. In both cases, an asymptotically valid confidence interval on the steady-state mean $\mu$ can be constructed by using the sample mean $\bar{X}_{n}$ and the sample variance of the replicate/batch means.

The trade-off between making a single long run (as in the BM method) and many independent replications (as in the IR method) has been studied by many authors in the simulation literature (see, e.g., Alexopoulos and Goldsman 2004, Argon and Andradóttir 2006, and references therein). An advantage of IR is that the replication means are independent; however, it may suffer from the presence of an initial transient at the beginning of each of the $r$ runs, thus resulting in highly biased estimators for $\mu$ and $\sigma^{2}$. On the other hand, with the BM method, initialization bias often exists only in the first batch of observations; however, the batch means are usually correlated. Most of the studies in the literature comparing these two methods conclude that the initial transient problem often renders IR too dangerous to use in steady-state simulations.

To combine the advantages of the BM and IR methods, Argon and Andradóttir (2006) propose a compromise - the method of replicated batch means (RBM) - in which a few independent replications are run, each containing the same number of batches. They point out that an added advantage in using multiple replications (as in IR and RBM) is that the individual replications can be started in different initial states, which can be used to ensure that observations from various different parts of the state space of the underlying process 
will be generated. This is especially important in the analysis of stochastic processes that have nearly decomposable state spaces. The analytical results by Argon and Andradóttir (2006) show that RBM typically has asymptotic performance characteristics which, not surprisingly, fall between those of IR and BM for stationary processes. However, their numerical results for non-stationary processes suggest that the RBM method, implemented with a good choice of initialization method and a small number of replications, provides confidence interval coverages (for $\mu$ ) that range from being similar to those of BM to being substantially better.

In a follow-up paper, the authors of this paper extend the analytical results obtained by Argon and Andradóttir (2006) to non-stationary stochastic processes with additive transient processes (see Alexopoulos et al. 2006). In particular, we obtain analytical results on the asymptotic performance of the RBM mean and variance estimators for stochastic processes that possess both stationary and transient components, with the transient components fading away as the number of observations grows. The current paper provides a review of the main results of Alexopoulos et al. (2006) and our ongoing work on this topic.

The outline of this paper is as follows. Section 2 provides definitions, assumptions, and results that we use in the remainder of the paper. Section 3 summarizes our main results for the mean and variance of the RBM variance estimator when the underlying stochastic process has an additive initial transient. Section 4 provides the main results of Alexopoulos et al. (2006), which are special cases of the results provided in Section 3. In Section 5, we provide an example to show how the results presented in Sections 3 and 4 can be used to obtain insights on the selection of the best number of replications for the replicated batch means method when the sample size is large. We provide our concluding remarks in Section 6. Since the proofs of our results are lengthy, they are omitted in this paper.

\section{PRELIMINARIES}

In this section, we state some definitions and assumptions.

Definitions. We conduct $r$ independent replications of the process $\left\{X_{k}\right\}$, each containing $n$ observations in addition to the initial state. Each replication is divided into $b$ nonoverlapping batches of $m$ observations (we assume that $n=m b$ and exclude the initial states of the replications from the batch means). Hence, the total number of batches is $r b$, and we assume throughout that $r b \geq 2$. Let $X_{i, k}$ denote observation $k$ from replication $i$, for $i=1,2, \ldots, r$ and $k=0,1,2, \ldots, n$ (so that $X_{i, 0}$ denotes the initial state of replication $i$ ). The $j$ th batch mean from the $i$ th replication is denoted by

$$
\bar{X}_{i, j, m} \equiv \frac{1}{m} \sum_{k=1}^{m} X_{i,(j-1) m+k}, \quad i=1,2, \ldots, r, j=1,2, \ldots, b ;
$$

the $i$ th replicate mean is

$$
\bar{X}_{i, n} \equiv \frac{1}{b} \sum_{j=1}^{b} \bar{X}_{i, j, m}=\frac{1}{n} \sum_{k=1}^{n} X_{i, k}, \quad i=1,2, \ldots, r ;
$$

and the overall sample mean is $\bar{X}_{\mathrm{RBM}}(r, n) \equiv \sum_{i=1}^{r} \bar{X}_{i, n} / r$. Finally, we define the replicated batch means estimator for $\sigma^{2}$ as

$$
\widehat{V}_{\mathrm{RBM}}(r, b, m)=\frac{m}{r b-1} \sum_{i=1}^{r} \sum_{j=1}^{b}\left(\bar{X}_{i, j, m}-\bar{X}_{\mathrm{RBM}}(r, n)\right)^{2} .
$$

Now suppose that $\left\{S_{k}\right\}$ is a stationary process. Following Billingsley (1968), let $\mathscr{M}_{\ell_{1}}^{\ell_{2}}\left(\ell_{1} \leq \ell_{2}\right)$ denote the sigma field generated by $\left\{S_{k}\right\}_{k=\ell_{1}}^{\ell_{2}}$. We say that $\left\{S_{k}\right\}$ is $\phi$-mixing if for all $j, \ell \geq 1$ and any events $A \in \mathscr{M}_{-\infty}^{j}$ and $B \in \mathscr{M}_{j+\ell}^{\infty}$, we have $|P(B \mid A)-P(B)| \leq \phi_{\ell}$, where $\phi_{\ell} \rightarrow 0$ as $\ell \rightarrow \infty$ and, without loss of generality, the $\phi_{\ell}$ are nonincreasing.

Let $\mathbf{v}=\left(v_{1}, \ldots, v_{k}\right)$ be a vector of dimension $k \geq 1$, $f: \mathbb{R}^{k} \rightarrow \mathbb{R}$ be a function, and $g: \mathbb{R}^{k} \rightarrow \mathbb{R}^{+}$be a positive function. Throughout the paper, the notation $f(\mathbf{v})=o(g(\mathbf{v}))$ means that $f(\mathbf{v}) / g(\mathbf{v}) \rightarrow 0$ as $v_{\ell} \rightarrow \infty$ for all $\ell \in\{1, \ldots, k\}$. We also define the relevant quantity $\gamma \equiv-2 \sum_{j=1}^{\infty} j R_{j}$, where $R_{j} \equiv \operatorname{Cov}\left(S_{1}, S_{1+j}\right)(j \geq 1)$ is the autocovariance function (see, e.g., Song and Schmeiser 1995).

We shall henceforth consider the following model and standing assumptions.

Assumption 1 The simulation output process $\left\{X_{i, k}\right\}_{k=1}^{\infty}$ from replication $i=1,2, \ldots, r$ can be represented in the form

$$
X_{i, k}=S_{i, k}+A_{i, k},
$$

$k=1,2, \ldots$, where $\left\{S_{i, k}\right\}_{k>1}$ is stationary and $\phi$-mixing with $\mathrm{E}\left[S_{i, 1}^{12}\right]<\infty$ and $\phi_{\ell}=O\left(\ell^{-9}\right)$, and $\left\{A_{i, k}\right\}$ is an initial transient process. Without loss of generality, we take $\mathrm{E}\left[S_{i, 1}\right]=0$ for all $i=1,2, \ldots, r$. (Of course, Assumption 1 accommodates the stationary case if all of the $A_{i, k}$ 's equal 0 .)

With model (1) in mind, the stationary and transient portions of the $j$ th batch mean from replication $i$, where $i=1,2, \ldots, r$ and $j=1,2, \ldots, b$, are

$$
\bar{S}_{i, j, m} \equiv \frac{1}{m} \sum_{k=1}^{m} S_{i,(j-1) m+k} \text { and } \bar{A}_{i, j, m} \equiv \frac{1}{m} \sum_{k=1}^{m} A_{i,(j-1) m+k},
$$


respectively; the stationary and transient portions of the $i$ th replicate mean, where $i=1,2, \ldots, r$, are

$$
\bar{S}_{i, n} \equiv \frac{1}{b} \sum_{j=1}^{b} \bar{S}_{i, j, m} \quad \text { and } \quad \bar{A}_{i, n} \equiv \frac{1}{b} \sum_{j=1}^{b} \bar{A}_{i, j, m}
$$

respectively; and the stationary and transient portions of the overall sample mean are $\bar{S}(r, n) \equiv \sum_{i=1}^{r} \bar{S}_{i, n} / r$ and $\bar{A}(r, n) \equiv$ $\sum_{i=1}^{r} \bar{A}_{i, n} / r$, respectively.

We will also need the following two assumptions.

Assumption 2 The transient processes $\left\{A_{i, k}\right\}_{k \geq 1}$ are independent of the stationary processes $\left\{S_{\ell, k}\right\}_{k \geq 1}$ for all $i, \ell=1,2, \ldots, r$. Furthermore, the transient processes $\left\{A_{i, k}\right\}_{k \geq 1}$ are independent and stochastically equivalent for all $i=1,2, \ldots, r$, and similarly, the stationary processes $\left\{S_{i, k}\right\}_{k \geq 1}$ are independent and stochastically equivalent for all $i=1,2, \ldots, r$.

Assumption 3 For all $i=1,2, \ldots, r$, $\max _{j \in\{1,2, \ldots, b\}} \mathrm{E}\left[\left(\bar{A}_{i, j, m}-\bar{A}_{i, n}\right)^{2}\right] \rightarrow 0 \quad$ as $m \rightarrow \infty$ and $b \rightarrow \infty$, and $\mathrm{E}\left[\left(\bar{A}_{i, n}-\bar{A}(r, n)\right)^{2}\right] \rightarrow 0$ as $n \rightarrow \infty$.

The following proposition provides sufficient conditions under which Assumption 3 is satisfied.

Proposition 1 Assumption 3 is satisfied if $\mathrm{E}\left[\bar{A}_{i, n}^{2}\right] \rightarrow$ 0 as $n \rightarrow \infty$ and $\mathrm{E}\left[\bar{A}_{i, j, m}^{2}\right] \rightarrow 0$ as $m \rightarrow \infty$, uniformly with respect to $j$, for all $i=1,2, \ldots, r$.

\section{MAIN RESULTS}

In this section, we provide results on the expected value and variance of the RBM estimator of the variance parameter $\sigma^{2}$ in the presence of a (stochastic) initial transient.

Theorem 1 Under Assumptions 1 and 2, we have

$$
\begin{aligned}
& \mathrm{E}\left[\widehat{V}_{\mathrm{RBM}}(r, b, m)\right]=\sigma^{2}+\frac{\left(r b^{2}-1\right) \gamma}{(r b-1) b m} \\
&+\frac{r m}{r b-1} \sum_{j=1}^{b} \mathrm{E}\left[\left(\bar{A}_{1, j, m}-\bar{A}(r, n)\right)^{2}\right]+o\left(\frac{1}{m}\right),
\end{aligned}
$$

as $m \rightarrow \infty$, where the $o(1 / m)$ term is uniform with respect to $b$.

Theorem 2 Under Assumptions 1-3, we have

$$
\begin{aligned}
r b \operatorname{Var}( & \left.\widehat{V}_{\mathrm{RBM}}(r, b, m)\right) \\
= & 2 \sigma^{4}+\frac{4 r^{2} b m \sigma^{2}}{(r b-1)^{2}} \sum_{j=1}^{b} \mathrm{E}\left[\left(\bar{A}_{1, j, m}-\bar{A}(r, n)\right)^{2}\right] \\
& +\frac{r^{2} b m^{2}}{(r b-1)^{2}} \sum_{j=1}^{b} \sum_{\ell=1}^{b} \operatorname{Cov}\left(\left(\bar{A}_{1, j, m}-\bar{A}(r, n)\right)^{2},\right. \\
& \left.\left(\bar{A}_{1, \ell, m}-\bar{A}(r, n)\right)^{2}\right)+o(1),
\end{aligned}
$$

\section{A SPECIAL TRANSIENT PROCESS}

In this section, we consider an interesting special case of our general model (1) where $A_{i, k}=\alpha a_{k}$ for all $k=$ $1,2, \ldots, n$ and $i=1,2, \ldots, r,\left\{a_{k}\right\}$ is a sequence of scalars satisfying $a_{k} \rightarrow 0$ as $k \rightarrow \infty$, and $\alpha$ is the deterministic initial state of the process. Hence, the transient process is assumed to be the same in all replications, and it is a deterministic sequence that converges to zero as the length of the simulation run increases. This special transient process (with $\alpha=1$ ) was used by Alexopoulos and Goldsman (2004) and Alexopoulos et al. (2006) to compare the batch means and independent replications methods, and to study the replicated batch means method, respectively. It models situations where all replications are started in the same initial state.

We first define some notation. For $j=1,2, \ldots, b$, let $\bar{a}_{j, m}=\sum_{k=(j-1) m+1}^{j m} a_{k} / m$, and $\bar{a}_{n}=\sum_{k=1}^{n} a_{k} / n$. It is easy to see that the transient process $\left\{A_{i, k}\right\}$ under consideration in this section satisfies Assumption 3 (see Proposition 1). We can now rewrite the results of Theorems 1 and 2 as follows:

$$
\begin{aligned}
& \mathrm{E}\left[\widehat{V}_{\mathrm{RBM}}(r, b, m)\right]=\sigma^{2}+\frac{\left(r b^{2}-1\right) \gamma}{(r b-1) b m} \\
& \quad+\frac{r m \alpha^{2}}{r b-1} \sum_{j=1}^{b}\left(\bar{a}_{j, m}-\bar{a}_{n}\right)^{2}+o\left(\frac{1}{m}\right), \text { as } m \rightarrow \infty,
\end{aligned}
$$

and

$$
\begin{aligned}
& r b \operatorname{Var}\left(\widehat{V}_{\mathrm{RBM}}(r, b, m)\right) \\
& =2 \sigma^{4}+\frac{4 r^{2} b m \sigma^{2} \alpha^{2}}{(r b-1)^{2}} \sum_{j=1}^{b}\left(\bar{a}_{j, m}-\bar{a}_{n}\right)^{2}+o(1),
\end{aligned}
$$

as $m \rightarrow \infty$ and $b \rightarrow \infty$, under Assumptions 1 and 2. Equations (4) and (5) are proved in Theorems 1 and 2, respectively, in Alexopoulos et al. (2006), for the case where $\alpha=1$.

\section{AN EXAMPLE}

In this section, we study a specific case of the transient process considered in Section 4 to determine the number of replications that minimizes the absolute asymptotic bias of the variance estimator when the total number of batches $B=r b$ is fixed. More specifically, we consider an exponentially decaying sequence, for which $a_{k}=a^{k}$ for some $-1<a<1$. Exponentially fast convergence to steady state holds for many stochastic processes as noted in Glynn and Heidelberger (1991). For example, autoregressive sequences and irreducible, finite-state, continuous-time Markov chains exhibit exponential convergence to steady state.

as $m \rightarrow \infty$ and $b \rightarrow \infty$. 
It is easy to see that for this exponentially decaying transient process, we have

$$
\bar{a}_{1, m}=\frac{a}{(1-a) m}+o\left(\frac{1}{m}\right) \text { as } m \rightarrow \infty .
$$

It is also easy to see that $\bar{a}_{j, m}=o(1 / m)$ as $m \rightarrow \infty$ for $j=2,3, \ldots, b$. Plugging these expressions and also $b=B / r$ into Equation (4), we obtain

$$
\begin{gathered}
\mathrm{E}\left[\widehat{V}_{\mathrm{RBM}}(r, B / r, m)\right]=\sigma^{2}+\frac{1}{B(B-1) m}\left[-\frac{a^{2} \alpha^{2}}{(1-a)^{2}} r^{2}\right. \\
\left.+\left(\frac{a^{2} B \alpha^{2}}{(1-a)^{2}}-\gamma\right) r+B^{2} \gamma\right]+o\left(\frac{1}{m}\right), \text { as } m \rightarrow \infty .
\end{gathered}
$$

From Equation (6), we can see that the most dominant term in the bias of the variance estimator is a concave function of $r$, and hence the minimizer of the absolute asymptotic bias of the variance estimator either will be around one of the roots or the extreme point of this function, or will be equal to 1 or $B$. This implies that depending on the values of $a, B, \alpha$, and $\gamma, r=1$ (as in the BM method), $r=B$ (as in the IR method), or $1<r<B$ (as in the strict RBM method) may minimize the absolute bias of the variance estimator.

To illustrate, we next study a numerical example in which we set $B=32$ and consider $r \in\{1,2,4,8,16,32\}$. Suppose that the stationary portion of the process is a first-order autoregressive process, $\operatorname{AR}(1)$, defined by $S_{k}=\phi S_{k-1}+\varepsilon_{k}$ for $k \geq 1$, where $S_{0} \sim N(0,1)$, the $\left\{\varepsilon_{k}\right\}_{k \geq 1}$ are independent $N\left(0,1-\phi^{2}\right)$ random variables that are independent of $S_{0}$, and $-1<\phi<1$. (Note that this $\operatorname{AR}(1)$ process is stationary with $S_{k} \sim N(0,1)$ for all $k \geq 1$.) The $\mathrm{AR}(1)$ process $\left\{S_{k}\right\}$ has covariance function $R_{k}=\phi^{|k|}$ for all $k=0, \pm 1, \pm 2, \ldots$, so that $\sigma^{2}=(1+\phi) /(1-\phi)$ and $\gamma=-2 \phi /(1-\phi)^{2}$; see, e.g., Sargent et al. (1992).

Suppose also that for the transient portion of the process $\left\{A_{k}\right\}_{k \geq 1}$, we have $a=\phi$. Then, we have

$$
X_{k}=A_{k}+S_{k}=\phi A_{k-1}+\phi S_{k-1}+\varepsilon_{k}=\phi X_{k-1}+\varepsilon_{k},
$$

where $A_{0}=\alpha$. This shows that $\left\{X_{k}\right\}_{k \geq 1}$ is a (non-stationary) $\mathrm{AR}(1)$ process, where $X_{0} \sim N(\alpha, 1)$. For such a process, we next determine the best number of replications that minimizes the absolute asymptotic bias of the variance estimator (see Equation (6)) for various values of $\alpha$ and $\phi$.

From Table 1, we observe that as $\phi$ increases (so that the transient decays at a slower rate), we should decrease the number of replications even though the autocorrelation among the data intensifies with an increasing value of $\phi$. Table 1 also shows that the best number of replications also depends heavily on the initial state of the process. In particular, if we have a rough idea as to what the mean of the process is (such as in the case where $\alpha=1$ ), then we should consider making more replications. Our ongoing research focuses on obtaining additional insights into how the number of replications $r$ should be chosen (e.g., for other transient processes with different convergence rates).

Table 1: The value of $r$ that minimizes the absolute bias of the RBM variance estimator for large $m$ under a nonstationary $\mathrm{AR}(1)$ process.

\begin{tabular}{||c||c|c|c||}
\hline$\phi$ & $\alpha=1$ & $\alpha=5$ & $\alpha=10$ \\
\hline \hline 0.1 & 32 & 16 & 8 \\
0.2 & 16 & 16 & 4 \\
0.3 & 16 & 16 & 2 \\
0.4 & 16 & 8 & 2 \\
0.5 & 16 & 8 & 1 \\
0.6 & 16 & 4 & 1 \\
0.7 & 16 & 4 & 1 \\
0.8 & 16 & 4 & 1 \\
0.9 & 16 & 4 & 1 \\
\hline
\end{tabular}

\section{CONCLUSIONS}

We studied the replicated batch means (RBM) method applied to steady-state simulations of stochastic processes with additive transient components. In particular, we provided asymptotic expressions for the expected value and variance of the RBM variance estimator for large sample sizes. Finally, we used a simple example in which the transient decays at an exponential rate to demonstrate how our results can be used to obtain insights on the selection of the best number of replications in steady-state simulations.

\section{ACKNOWLEDGMENTS}

The work of the second author was supported by the National Science Foundation under Grant No. 0217860 and Grant No. 0400260. The work of the third author was supported by the National Science Foundation under Grant No. 0715020. The work of the fourth author was supported by the National Science Foundation under Grant No. 0400260.

\section{REFERENCES}

Alexopoulos, C., S. Andradóttir, N. T. Argon, and D. Goldsman. 2006. Replicated batch means variance estimators in the presence of an initial transient. ACM Transactions on Modeling and Computer Simulation 16 (4): 317-328.

Alexopoulos, C., and D. Goldsman. 2004. To batch or not to batch? ACM Transactions on Modeling and Computer Simulation 14 (1): 76-114. 
Argon, N. T., and S. Andradóttir. 2006. Replicated batch means for steady-state simulations. Naval Research Logistics 53 (6): 508-524.

Billingsley, P. 1968. Convergence of Probability Measures. New York: John Wiley and Sons.

Fishman, G. S. 2001. Discrete-event Simulation: Modeling, Programming, and Applications. New York: SpringerVerlag.

Glynn, P. W., and P. Heidelberger. 1991. Analysis of initial transient deletion for replicated steady-state simulations. Operations Research Letters 10 (8): 437-443.

Sargent, R. G., K. Kang, and D. Goldsman. 1992. An investigation of finite-sample behavior of confidence interval estimators. Operations Research 40 (5): 898913.

Song, W.-M. T., and B. W. Schmeiser. 1995. Optimal mean-squared-error batch sizes. Management Science 41 (1): 110-123.

\section{AUTHOR BIOGRAPHIES}

CHRISTOS ALEXOPOULOS is an Associate Professor in the School of Industrial and Systems Engineering at the Georgia Institute of Technology. His research interests are in the areas of simulation, statistics, and optimization of stochastic systems. He is a member of INFORMS and an active participant in the Winter Simulation Conference, having been Proceedings Co-Editor in 1995, Associate Program Chair in 2006, and a member of the Board of Directors since 2007. He is also the Simulation Department Editor of IIE Transactions. His e-mail address is <christoseisye.gatech.edu>, and his web page is <www.isye.gatech. edu/ christos>.

SIGRÚN ANDRADÓTTIR is a Professor in the H. Milton Stewart School of Industrial and Systems Engineering at the Georgia Institute of Technology. Previously, she was an Assistant Professor and later an Associate Professor in the Departments of Industrial Engineering, Mathematics, and Computer Sciences at the University of Wisconsin - Madison. She received her Ph.D. in Operations Research from Stanford University in 1990. Her research interests include simulation, applied probability, and stochastic optimization. She is a member of INFORMS and served as Editor of the Proceedings of the 1997 Winter Simulation Conference. She is the Simulation Area Editor of Operations Research Letters and has served as Associate Editor for various journals. Her e-mail address is <a@isye.gatech.edur, and her web page is <http://www.isye.gatech. edu/faculty/sa>.

NILAY TANIK ARGON is an Assistant Professor in the Department of Statistics and Operations Research at the University of North Carolina at Chapel Hill. She received her Ph.D. degree in Industrial Engineering in 2002 from the Georgia Institute of Technology. Her primary research interests include the modeling and analysis of stochastic systems and statistical simulation output analysis. She is a member of INFORMS. Her e-mail address is <nilay@unc.edu>, and her web page is $\langle$ http://www. unc. edu/ nilay $>$.

DAVID GOLDSMAN is a Professor in the H. Milton Stewart School of Industrial and Systems Engineering at the Georgia Institute of Technology. He received his Ph.D. in Operations Research and Industrial Engineering from Cornell University. His research interests include simulation output analysis and ranking and selection. $\mathrm{He}$ is an active participant in the Winter Simulation Conference, having been Program Chair in 1995, and having served on the WSC Board of Directors since 2002. His e-mail address is <smanlgatech.edu>, and his web page is <http: //www. isye.gatech. edu/ sman $>$. 